\title{
Constraining the location of gamma-ray emission in blazar jets
}

\section{High-energy emission in Blazars}

\author{
Manasvita Joshi ${ }^{1, a}$, Alan Marscher ${ }^{1}$, and Markus Böttcher ${ }^{2}$ \\ ${ }^{1} 725$ Commonwealth Ave., CAS 506, Boston University, Boston, MA - 02215 \\ ${ }^{2}$ Centre for Space Research, North-West University, Potchefstroom Campus, Potchefstroom 2520, South Africa
}

\begin{abstract}
.
The location of $\gamma$-ray emission in blazar jets has remained elusive as wetry to understand jet emission despite the extensive multiwavelength campaigns and vigorous theoretical efforts to understand the multiwavelength spectra. The synergy between multiwavelength campaigns and VLBA studies has resulted in correlation between the majority of $\gamma$-ray events and disturbances propagating down the parsec-scale jet. This implies that the $\gamma$-ray emission might originate beyond the broad line region (BLR), perhaps on scales comparable to the size of the dusty torus. On the other hand, external Compton models in which $\gamma$-ray emission is limited to sites inside the BLR have been used to explain the high-energy emission of many blazars. Thus, comprehending the time-dependent impact of all the three external components of seed photon field, namely the accretion disk, the BLR, and the dusty torus, on the evolution of the spectral energy distribution (SED) can be used as an important tool for connecting the origin of $\gamma$-ray emission of a flare to its multiwavelength properties.

Here, we use a multi-zone time-dependent leptonic jet model, with radiation feedback, to address this aspect of blazar jet emission. We let the system evolve to beyond the BLR and within the dusty torus. We explore the effects of varying the contribution of the disk, the BLR, and the dusty torus on the resultant seed photon field and their manifestation on the simulated SED of a typical blazar to gain insight on the location of the $\gamma$-ray emission region.
\end{abstract}

\section{Introduction}

Blazars are known for their highly variable emission across the electromagnetic spectrum. Their spectral energy distribution (SED) and variability patterns can be used as key features in deciphering the nature of the particle population, acceleration of particles, and the environment around the jet that is responsible for the observed emission. Conversely, modeling of such observational features requires inclusion of the nature of the particle population and the jet environment, as accurately as possible, in order to reach a better agreement between theoretical and observational results.

The SED of a blazar consists of two broad bumps [1], where the first bump is associated with the synchrotron process and the second is attributed to inverse Comptonization (IC) of photons by the same electrons producing synchrotron photons. The seed photon field for the IC process could either be due to synchrotron self-Compton (SSC) [2] and/or external Compton (EC) [3] processes. As far as $\gamma$-ray emission is concerned, it is always associated with IC processes, but can be entirely due to SSC, a combination of SSC and EC, or entirely due to EC, depending on the type and state of the blazar being observed.

Traditionally, the $\gamma$-ray emission has been thought to originate at sub-pc scales from within the broad line re-

\footnotetext{
a e-mail: mjoshi@bu.edu
}

gion (BLR), keeping the range of the location to between 0.01 and $0.3 \mathrm{pc}$ [4-6]. This was suggested to explain the intra-day variability timescales observed in $\gamma$-ray flares, which seemed to indicate that the emission region could not be too far away from the central engine on the basis of light crossing timescales [6,7]. At the same time, the emission region could not be too close to the central engine either due to constraints placed by the $\gamma-\gamma$ absorption process $[6,8]$. In addition, the strong dependence of the scattered flux on the level of boosting and the energy of incoming photons [9] also hinted at the emission region being located closer to the central source. This made the region within the BLR most favorable for the location of $\gamma$-ray emission. However, in the recent past, coincidences of $\gamma$-ray outbursts with radio events on pc scales have been observed [10,11], which seem to suggest a cospatial origin of radio and $\gamma$-ray events located at pc scales. As a result, it is argued that the $\gamma$-ray emitting region could also lie outside of the BLR [12, 13].

Thus, in order to understand the origin of $\gamma$-ray emission, it is important to let the system evolve to beyond the BLR into the dusty torus (DT) and include its contribution toward $\gamma$-ray emission so as to obtain a complete picture of all the seed photon fields responsible for high-energy emission in blazars. The goal of our current study is to understand how the $\gamma$-ray emission depends on the com- 
bination of various intrinsic physical parameters. We explore this aspect by including various components of seed photon fields in order to understand their effects on the dynamic evolution of SEDs.

\section{Seed Photon Fields}

To carry out this study, we use the multi-zone timedependent leptonic jet model, with radiation feedback scheme, of Joshi \& Böttcher (2011) (hereafter Paper I) in the internal shock model scenario. We consider three sources of seed photon fields, namely the accretion disk (D), the BLR, and the DT, and extend the model of Paper I to include the EC processes related to each source: ECD, ECBLR, and ECDT. At the start of our simulation the emission region is located closer to the accretion disk. We start following the evolution of the system, in a timedependent manner, as it moves outside the BLR and into the DT region. We consider anisotropic seed photon field from each of these three components, which is important for correctly accounting for their contribution in producing high-energy emission in blazars. In the following, we briefly describe the geometry and the anisotropic field of each of the three sources of target radiation field.

We consider a multi-color, geometrically thin, and optically thick accretion disk based on the model of Shakura \& Sunyaev (1973) [14]. Figure 1 represents a schematic of the disk geometry and the angular dependence of the spectral flux from the disk on the position of the emission region in the jet. We assume that the disk extends from $R_{\text {in,disk }}$ to $R_{\text {out,disk }}$ with the dimensionless energy, $\epsilon_{\mathrm{D}}$, of the incoming disk photon typically peaking in the UV in the lab frame. Here and in subsequent sections, dimensionless energy has been defined as, $\epsilon=\frac{h v}{m_{e} c^{2}}$. All the unprimed quantities refer to the AGN (lab) frame, whereas the primed ones stand for the comoving (plasma) frame.

As far as the BLR is concerned, we model it as a geometrically thick spherical shell extending from the inner, $R_{\text {in,BLR }}=0.2 \mathrm{pc}$, to outer, $R_{\text {out }, \mathrm{BLR}}=0.599 \mathrm{pc}$, radius and emitting in the energy range from infrared (IR) to soft Xrays such that $\epsilon_{\mathrm{BLR}}$ typically peaks in the UV. We take into account both the broad line emission and the diffuse continuum radiation [8] to provide the source of target photon field for the ECBLR process. Figure 2 shows the BLR geometry under consideration and the three positions for which the anisotropic radiation field has been calculated. Figure 3 shows the anisotropic profile of intensity obtained for these three positions.

A clumpy molecular torus [12] is considered to model the contribution of this source in producing $\gamma$-ray emission [see, e.g., 19]. The torus is assumed to be dominated by dust radiating as a blackbody in the IR at a temperature $\mathrm{T}=1200 \mathrm{~K}[16]$. The energy of the incoming photons, $\epsilon_{\mathrm{DT}}$, peaks in the IR. Figure 4 shows the schematic of the torus geometry under consideration. As can be seen from the figure, the torus lies in the plane of the accretion disk and extends from $R_{\mathrm{in}, \mathrm{DT}}=1 \mathrm{pc}$ to $R_{\mathrm{out}, \mathrm{DT}}=2 \mathrm{pc}$. We assume that the incident radiation comes from the middle of the torus and is bracketed by the two angle limits, a

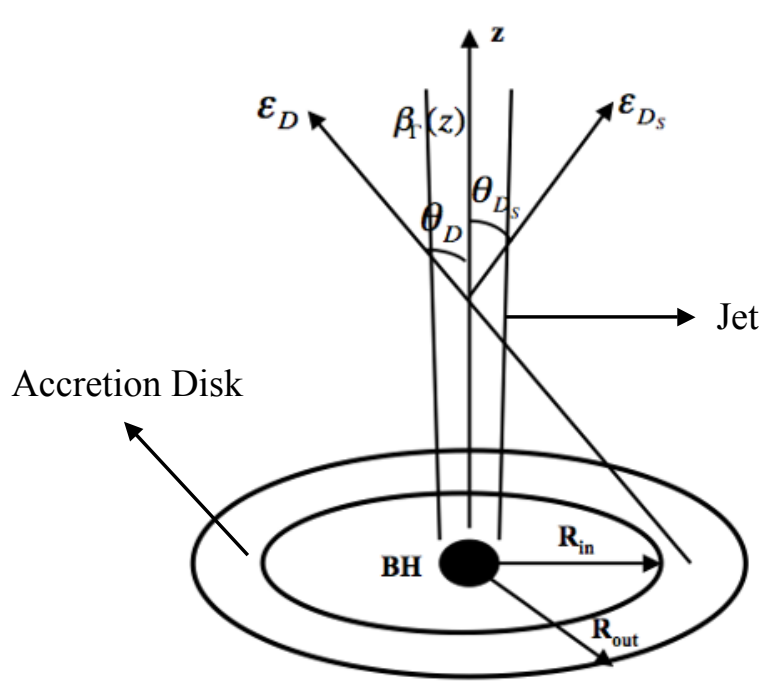

Figure 1. Schematic of the disk-jet geometry used in our model The disk temperature is calculated for all radii between $R_{\text {in,disk }}=$ $6.235 \times 10^{-5} \mathrm{pc}$ and $R_{\text {out }, \text { disk }}=0.058 \mathrm{pc}$. The emission region is located inside the jet and moves with velocity $\beta_{\Gamma_{\mathrm{sh}}} c[15]$ along the $\mathrm{z}$-axis (jet-axis). The incoming photon of dimensionless energy $\epsilon_{\mathrm{D}}$ from the disk intercepts the emitting volume at an angle $\theta_{\mathrm{D}}$ and the resulting outgoing photon with energy $\epsilon_{\mathrm{D}_{\mathrm{S}}}$ is scattered at an angle $\theta_{\mathrm{DS}_{\mathrm{S}}}$.

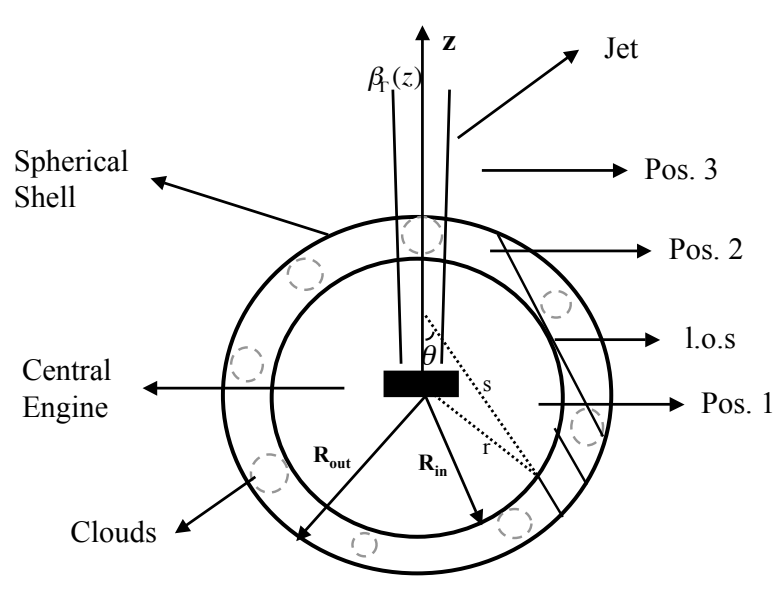

Figure 2. Schematic of the BLR geometry considered for the calculation of the EC component. Three positions of the emission region are considered, namely: (Pos.1) - region is located in the cavity of the BLR, (Pos.2) - region is located within the BLR, and (Pos.3) - region is located outside the BLR. The incoming photon makes an angle $\theta$ with the jet axis. Different lines of sight are shown that are calculated using the law of cosines between $r, s$ and $\theta$ for each of the three positions.

minimum $\theta_{\mathrm{DT}, \min }(z)$, and a maximum $\theta_{\mathrm{DT} \text {,max }}(z)$, which in turn are a function of distance $(\mathrm{z})$ along the jet-axis. As the emission region moves along the jet-axis, it sees only a fraction of this radiation, where the fraction is governed by the covering factor of the torus. 

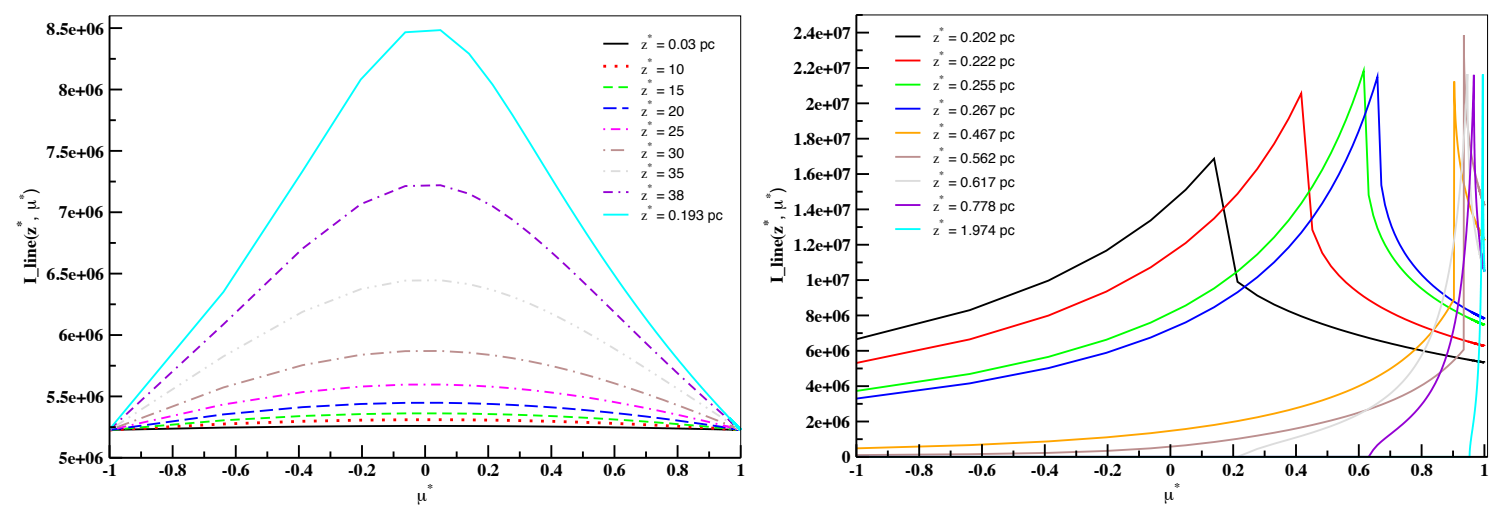

Figure 3. Plot of the intensity of radiation as a function of $\mu^{*}=\cos \theta^{*}$, due to BLR line emission. Here, the starred quantities in the graph refer to the lab frame. Top: Intensity profile for Pos.1, which is symmetric due to equal contribution of all lines of sight from the BLR. The profile peaks as the emission region reaches very close to the inner radius of the BLR. Bottom: Intensity profile for Pos. 2 and 3, which is asymmetric because unequal lines of sight contribute to the intensity calculation. The intensity distribution peaks at Pos. 2 when the emission region is located within the BLR shell, at $\sim 0.562 \mathrm{pc}$. The intensity plummets as the emission region emerges from the BLR shell, at $\sim 0.617 \mathrm{pc}$, and stays constant thereafter.

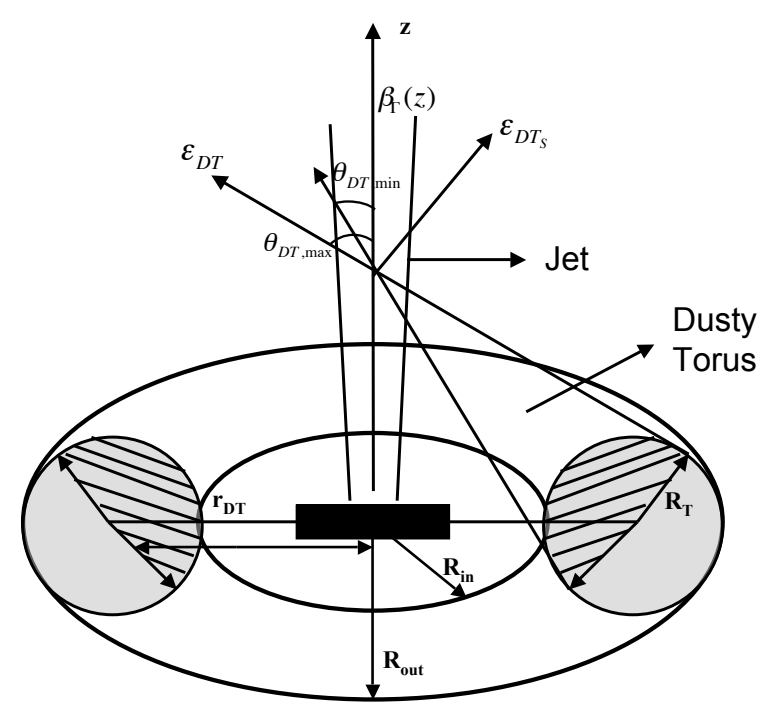

Figure 4. Model for the DT geometry used in ECDT calculations. The central circle of the torus is located at a distance $r_{\mathrm{DT}}=\frac{R_{\mathrm{out}, \mathrm{DT}}+R_{\mathrm{in}, \mathrm{DT}}}{2}$ from the central engine. The shaded portion shows the cross-sectional area of the torus with radius $R_{\mathrm{T}}=\frac{R_{\mathrm{out}, \mathrm{DT}}-R_{\mathrm{in}, \mathrm{DT}}}{2}$. Only a fraction of the torus facing the central continuum source (region with solid lines) is assumed to be hot enough to be visible to the emission region. The incoming photons, from this portion of the surface, enter the jet at angles whose range is governed by a minimum, $\theta_{\mathrm{DT} \text {, min }}(z)$, and a maximum, $\theta_{\mathrm{DT}, \max }(z)$ angle. The dimensionless energy of an incoming photon is given by $\epsilon_{\mathrm{DT}}$ while that of an outgoing photon is given by $\epsilon_{\mathrm{DT}, \mathrm{S}}$.

\section{Results}

We have run 8 simulations to understand the effects of varying a physical parameter related to the EC process on the evolution of the SED of a generic blazar. The value of each of the relevant parameters was varied two to three times to study its effect on the overall flux level of the simulated SED. As mentioned in Paper I, the unprimed quantities refer to the AGN frame, primed quantities to the comoving frame, and starred quantities indicate the observer's frame.

Table 1 shows the values of the base set (run 1) parameters used to obtain the baseline model. The parameters of this generic blazar are motivated by a fit to the blazar 3C 279. The input parameters up to $\theta_{\text {obs }}^{*}$ have been explained in Paper I. The left side of Fig. 5 shows the resultant time-integrated SED of the baseline model for a generic blazar source, over a 1-day flaring period. According to the model description given in Paper I, the input parameters for the base set are used to analytically obtain values for the BLF of the emission region $\Gamma_{\mathrm{sh}}=14.9$, and magnetic field $B^{\prime}=2.71 \mathrm{G} \& \gamma_{\max }^{\prime}=1.26 \times 10^{5}$ for both the forward and reverse emission regions. On the other hand, the value of $\gamma_{\text {min }}^{\prime}$ for the forward emission region is numerically obtained to be $8.81 \times 10^{2}$ and that for the reverse emission region is $1.85 \times 10^{3}$. Similarly, the total width of the forward and reverse emission region is analytically obtained to be $\Delta_{\mathrm{fs}}^{\prime}=1.01 \times 10^{16} \mathrm{~cm}$ and $\Delta_{\mathrm{rs}}^{\prime}=2.12 \times 10^{16} \mathrm{~cm}$, with the shock crossing time for each of the emission regions being $t_{\mathrm{cr}, \mathrm{fs}}^{\prime}=8.93 \times 10^{5} \mathrm{~s}$ and $t_{\mathrm{cr}, \mathrm{rs}}^{\prime}=1.37 \times 10^{6} \mathrm{~s}$. The inner and outer shells collide at a distance of $z=1.01 \times 10^{17} \mathrm{~cm}$, making it the starting position of the emission region along the jet axis. The entire simulation runs for a total of $\sim 5$ days, in the observer's frame, in which the emission region moves beyond the BLR and into the DT, covering a distance of 1.04 pc. The flaring period of our simulation, in the observer's frame, corresponds to the time taken by forward and reverse shocks, in the comoving frame, to individually exit their respective emission regions (see Paper 1 for more details). For our baseline model, the forward shock exits the forward emission region within a day when the emission region is located in the cavity of the BLR, at $\sim 0.161 \mathrm{pc}$. Similarly, the reverse shock exits its region within a day when the emission region is located within the BLR, at 
$\sim 0.235 \mathrm{pc}$. The time of exit of shocks plays an important role in deciding the overall flux level of $\gamma$-ray emission. This is because irrespective of the location of the emission region with respect to the seed photon fields, the absence of shocks from the system signifies dominance of cooling which in turn affects the $\gamma$-ray emission from a blazar.

Table 2 lists the values of each of the parameters that are varied in the rest of the simulations. We study the effect on the simulated SED with respect to that of the baseline model. The right side of Fig. 5 shows the effect of varying the starting position of the emission region, along the jet axis, on the time-integrated SED of a generic blazar. In the case of the base set (run 1), the ECBLR process dominates over the rest of the EC processes and peaks at $\sim 0.4 \mathrm{GeV}$. The ECDT process is responsible for the emission at $\sim 40$ $\mathrm{MeV}$ and dominates over ECD and SSC processes. As can be seen from the figure, ECBLR dominates over other IC processes in producing the high-energy emission of the blazar as long as the emission region is located within the BLR (runs 1, 2, and 3). In the case of run 2, where the emission region is located much closer to the central engine, the ECD process overtakes the ECDT process but is still below the contribution from the ECBLR process. On the other hand, for the case where the emission region is placed outside the BLR (run 4), both DT and SSC processes dominate over the rest of the IC processes in producing $\gamma$-ray emission.

Figure 6 shows the effect of varying the covering factor of the BLR (left) and that of the DT (right) on the timeintegrated SED of the generic blazar with respect to the baseline model. The ECBLR component dominates over the rest of the IC components when its covering factor is increased. This increases the overall cooling in the system due to which the synchrotron component decreases and the Compton dominance (ratio of EC over synchrotron component) becomes much higher compared to run 1 . In the case of runs $7 \& 8$, the low-energy component of the simulated SEDs is similar to that of run 1. The high-energy component, on the other hand, starts to become dominated by the ECDT process as the covering factor for the DT is increased (run 8).

\section{Discussion and Conclusion}

We have extended the multi-zone time-dependent leptonic jet model, with radiation feedback scheme, in the internal shock scenario, of Joshi \& Böttcher (2011) to include the EC component by considering anisotropic target radiation fields. We consider three sources of seed photon fields, namely the accretion disk, the BLR, and the DT, and let the system evolve to beyond the BLR and into the DT in a time-dependent manner.

We have carried out a parameter study to understand the effects of varying input parameters relevant to the EC emission on the dynamic evolution of the SED of a generic blazar. The results clearly show the importance of considering an anisotropic target radiation field, as that enables the system to evolve beyond the BLR and let the contribution of each of the three components be incorporated as accurately as possible. For the cases considered here, in addition to the BLR, the DT plays an important role in contributing toward $\gamma$-ray emission and is responsible for the emission in the $\mathrm{MeV}$ range. Thus, understanding of the sources of seed photons is imperative in order to comprehend the high-energy emission of blazars in general. At the same time, it is important to realize that these three conventional sources might not be the complete answer to the question of correlation between $\gamma$-ray and radio events at pc scales that we are currently facing. In that case, tinkering with the possibility of new sources of seed photon fields or getting creative with the existing ones [see, e.g., $17,18,20]$ should be and needs to be explored. In addition to the position of the emission region along the jet axis, another factor that plays an important role in deciding the range of the location of $\gamma$-ray emission is the duration over which the shocks stay inside the system. This is because $\gamma$-ray emission is produced by highly energetic leptons, which, in turn, are produced in the system for as long as the shocks are present and accelerating particles to such high energies.

Therefore, understanding of the combination of the factors discussed above and how this affects the evolution of the system, in a time-dependent manner, is crucial to understanding the origin of $\gamma$-ray emission and its re-

Table 1. Parameter list of run 1 used to obtain the baseline model.

\begin{tabular}{lll}
\hline Parameter & Symbol & Value \\
\hline Kinetic Luminosity & $L_{w}$ & $5 \times 10^{47} \mathrm{erg} / \mathrm{s}$ \\
Event Duration & $t_{w}$ & $10^{7} \mathrm{~s}$ \\
Outer Shell Mass & $M_{o}$ & $1.531 \times 10^{32} \mathrm{~g}$ \\
Inner Shell BLF & $\Gamma_{i}$ & 26.3 \\
Outer Shell BLF & $\Gamma_{o}$ & 10 \\
Inner Shell Width & $\Delta_{i}$ & $6.2 \times 10^{15} \mathrm{~cm}$ \\
Outer Shell Width & $\Delta_{o}$ & $7.4 \times 10^{15} \mathrm{~cm}$ \\
Inner Shell Position & $z_{i}$ & $7.8 \times 10^{15} \mathrm{~cm}$ \\
Outer Shell Position & $z_{o}$ & $1.56 \times 10^{16} \mathrm{~cm}$ \\
Electron Energy Equipartition & $\varepsilon_{e}^{\prime}$ & $9 \times 10^{-2}$ \\
Magnetic Energy Equipartition & $\varepsilon_{B}^{\prime}$ & $2.5 \times 10^{-3}$ \\
Fraction of Accelerated Electrons & $\zeta_{e}^{\prime}$ & $1 \times 10^{-2}$ \\
Acceleration Timescale Parameter & $\alpha^{\prime}$ & $2 \times 10^{-5}$ \\
Particle Injection Index & $q^{\prime}$ & 4.0 \\
Slice/Jet Radius & $R_{z}^{\prime}$ & $5.44 \times 10^{16} \mathrm{~cm}$ \\
Observer Frame Observing Angle & $\theta_{\text {obs }}^{*}$ & $1.5 \mathrm{deg}$ \\
Disk Luminosity & $L_{\mathrm{disk}}$ & $8 \times 10^{45} \mathrm{erg} / \mathrm{s}$ \\
BH Mass & $M_{\mathrm{BH}}$ & $2 \times 10^{8} M_{\odot}$ \\
Accretion Efficiency & $\eta_{\text {acc }}$ & 0.06 \\
BLR Luminosity & $L_{\mathrm{BLR}}$ & $8 \times 10^{44} \mathrm{erg} / \mathrm{s}$ \\
BLR inner radius & $R_{\text {in,BLR }}$ & $6.17 \times 10^{17} \mathrm{~cm}$ \\
BLR outer radius & $R_{\text {out,BLR }}$ & $1.85 \times 10^{18} \mathrm{~cm}$ \\
BLR optical depth & $\tau_{\mathrm{BLR}}$ & 0.01 \\
BLR covering factor & $f_{\text {cov,BLR }}$ & 0.03 \\
DT inner radius & $R_{\text {in,DT }}$ & $3.086 \times 10^{18} \mathrm{~cm}$ \\
DT outer radius & $R_{\text {out,DT }}$ & $6.17 \times 10^{18} \mathrm{~cm}$ \\
Ldisk fraction & $L_{\mathrm{disk}, \mathrm{rac}}$ & 0.2 \\
DT covering factor & $f_{\text {cov,DT }}$ & 0.2 \\
Redshift & $Z^{*}$ & 0.538 \\
\hline & & \\
& & \\
\hline
\end{tabular}



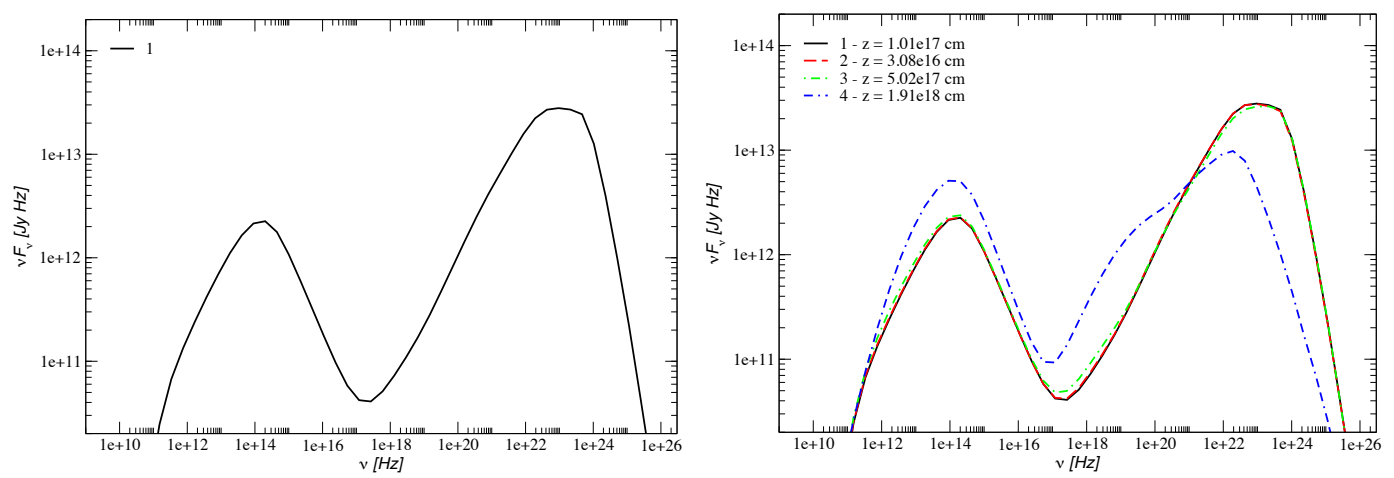

Figure 5. Left: Simulated SED of the baseline model of a generic blazar, time-integrated over a period of 1day. The EC component peaks in the $\gamma$-ray regime at $\sim 10^{23} \mathrm{~Hz}$ while the synchrotron component peaks at $\sim 10^{14} \mathrm{~Hz}$. The EC emission of the base set is dominated by the ECBLR component, which peaks at $\sim 0.4 \mathrm{GeV}$. The ECD component does not contribute to the high-energy component of this blazar while the ECDT component is responsible for the emission at $\sim 40 \mathrm{MeV}$. Right: Simulated SEDs of runs 2,3 , and 4 in which the starting position $(\mathrm{z})$ of the emission region along the jet axis was varied. As can be seen, the BLR continues to dominate as long as the emission region is located somewhere close to or within the BLR (run $2 \& 3$ ). In case of run 2, the ECD component takes over the dominance of the ECDT component at $\sim 40 \mathrm{MeV}$, but the $\gamma$-ray regime at $0.4 \mathrm{GeV}$ continues to be dominated by the ECBLR process. On the other hand, for the case where the starting position of the emission region is located outside of the BLR (run 4), the entire high-energy emission is dominated by the combination of DT and SSC processes.
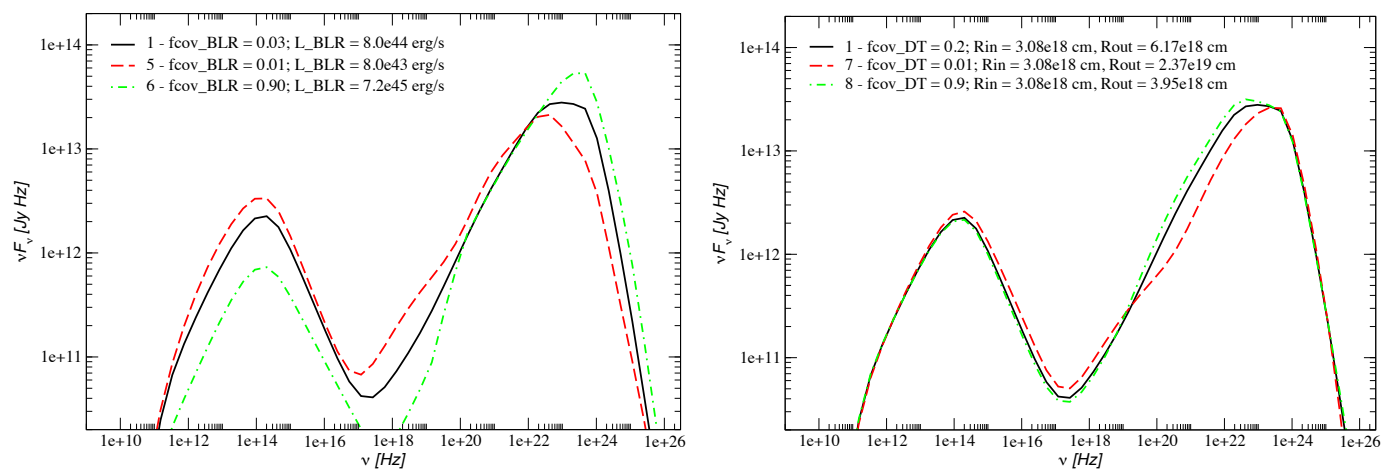

Figure 6. Left: Simulated time-integrated SEDs of runs $5 \& 6$ depicting the effect of varying the BLR covering factor and consequently its luminosity $\left(L_{\mathrm{BLR}} \sim f_{\text {cov,BLR }} L_{\text {disk }}\right)$. As can be seen, the ECBLR component becomes the most dominant as the covering factor increases and due to excessive cooling the corresponding synchrotron component goes down, making the Compton dominance higher for such a case. There is no significant shift in the location of the peaks of the low- and high-energy components in either of the cases. Right: Simulated SEDs of runs $7 \& 8$ showing the effect of changing the covering factor of the DT. In this case, as the contribution of DT increases with the corresponding covering factor (run 8), the $\gamma$-ray emission becomes dominated by the ECDT process.

Table 2. Parameter list for other simulations.

\begin{tabular}{ll}
\hline Run & Parameter Value \\
\hline 2 & $z=3.1 \times 10^{16} \mathrm{~cm}$ \\
3 & $z=5.0 \times 10^{17} \mathrm{~cm}$ \\
4 & $z=1.9 \times 10^{18} \mathrm{~cm}$ \\
5 & $f_{\text {cov,BLR }}=0.01$ \\
6 & $f_{\text {cov,BLR }}=0.9$ \\
7 & $f_{\text {cov,DT }}=0.01$ \\
8 & $f_{\text {cov,DT }}=0.9$ \\
\hline
\end{tabular}

lationship with emission at the lower energy end of the spectrum.

This research was supported by NASA through Fermi grants NNX11AQ03G and NNX12AO59G.

\section{References}

[1] G. Fossati, L. Maraschi, A. Celotti, A. Comastri, \& G. Ghisellini, MNRAS, 299, 433 (1998)

[2] S.D. Bloom, \& A.P. Marscher, ApJ, 461, 657 (1996)

[3] G. Ghisellini, A. Celotti, G. Fossati, L. Maraschi, \& A. Comastri, MNRAS, 301, 451 (1998)

[4] C.D. Dermer, \& R. Schlickeiser, ApJS, 90, 945 (1994)

[5] R.D. Blandford, \& A. Levinson, ApJ, 441, 79 (1995)

[6] G. Ghisellini, \& P. Madau, MNRAS, 280, 67 (1996)

[7] G. Ghisellini, \& F. Tavecchio, MNRAS, 397, 985 (2009)

[8] H.T. Liu, \& J.M. Bai, ApJ, 653, 1089 (2006)

[9] M. Sikora, M.C. Begelman, \& M.J. Rees, ApJ, 421, 153 (1994) 
[10] S.G. Jorstad, A.P. Marscher, J.R. Mattox, M.F. Aller, H.D. Aller, A.E. Wehrle, \& S.D. Bloom, ApJ, 556, 738 (2001)

[11] J. León-Tavares, E. Valtaoja, P. Giommi, G. Polenta, M. Tornikoski, A. Lähteenmäki, D. Gasparrini, \& S. Cutini, ApJ, 754, 23 (2012)

[12] A. Sokolov, \& A.P. Marscher, ApJ, 629, 52 (2005)

[13] E.J. Lindfors, E. Valtaoja, \& M. Türler, A\&A, 440, 845 (2005)

[14] M. Boettcher, H. Mause, \& R. Schlickeiser, A\&A, 324, 395 (1997)
[15] M. Joshi, \& M. Böttcher, ApJ, 727, 21 (2011)

[16] M.P. Malmrose, A.P. Marscher, S.G. Jorstad, R. Nikutta, \& M. Elitzur, ApJ, 732, 116 (2011)

[17] B.E. Stern, \& J. Poutanen, MNRAS, 417, L11 (2011)

[18] J. León-Tavares, V. Chavushyan, V. PatiñoÁlvarez, E. Valtaoja, T.G. Arshakian, L.Č. Popović, M. Tornikoski, A. Lobanov, A. Carramiñana, L. Carrasco et al., ApJL, 763, L36 (2013)

[19] A.P. Marscher, submitted to ApJ (2013)

[20] A.P. Marscher, ArXiv e-prints (2013), 1304. 2064 